\title{
Ökonomische Desinformation - Ursachen und Handlungsempfehlungen
}

\author{
In einer Demokratie ist eine gut informierte Bevölkerung besonders wichtig. In den Perils-of- \\ Perception-Studien wird untersucht, wie gut die deutsche Bevölkerung über wirtschaftliche \\ Fakten informiert ist. Dabei sind die Fehlwahrnehmungen groß und Deutschland rangiert im \\ Mittelfeld der fortgeschrittenen Volkswirtschaften. Es gibt verschiedene mögliche Erklärungen \\ für das relativ geringe Faktenwissen der deutschen Gesellschaft. Daraus lassen sich \\ Maßnahmen entwickeln, die dazu beitragen können, den wirtschaftlichen Informationsstand \\ zu verbessern. Diese Maßnahmen richten sich an verschiedene Beteiligte, wie politische \\ Entscheidungsträger:innen, die Medien und die akademische Gemeinschaft.
}

\begin{abstract}
Debatten über die „richtige“ Wirtschafts- und Reformpolitik werden nicht nur in Wahlkampfzeiten kontrovers geführt. Solche Kontroversen sind ein unverzichtbares Element der demokratischen Meinungsbildung. Objektiv richtige Lösungen kann es dabei nicht immer geben, denn die Antworten auf Fragen wie „Wieviel staatliche Umverteilung ist nötig?" oder „In welcher Art und in welchem Ausmaß sind regulative Eingriffe in Märkte notwendig?" hängen auch von subjektiven Werturteilen ab. Öffentliche Debatten, die viele Fakten beleuchten und auch Werturteile transparenter machen, können im Idealfall dabei helfen, sich wohlinformiert in den vielfältigen Zielkonflikten unserer Zeit zu positionieren. Aus gesellschaftlicher Sicht besteht dann ein Problem, wenn die Präferenzbildung der Wähler:innen durch mangelndes Wissen um wirtschaftspolitische Fakten und Zusammenhänge oder gar durch systematisch verzerrte Wahrnehmungen beeinflusst wird, denn durch systematische Informationsdefizite können die ökologischen, ökonomischen und finanziellen Restriktionen unserer Gesellschaft nicht angemessen beurteilt werden. Möglicherweise unterbleiben aufgrund solcher Defizite auch Reformen oder werden kostspielig verzögert (Caplan, 2007).*
\end{abstract}

\section{Informationsdefizite im internationalen Vergleich}

Wie informiert sind die Deutschen bezüglich wirtschaftspolitischer Themen auch im Vergleich mit anderen Ländern? Zur Beantwortung dieser Fragen wird auf die Perils-of-Percep-

(C) Der/die Autor:in 2021. Open Access: Dieser Artikel wird unter der Creative Commons Namensnennung 4.0 International Lizenz veröffentlicht (creativecommons.org/licenses/by/4.0/deed.de).

Open Access wird durch die ZBW - Leibniz-Informationszentrum Wirtschaft gefördert.

* Dieser Beitrag beruht auf dem Gutachten „Informationsdefizite als Hindernis rationaler Wirtschaftspolitik: Ausmaß, Ursachen und Gegenstrategien", das von der Brigitte Strube Stiftung unterstützt wurde.
tion-(PoP)-Umfragen zurückgegriffen (Ipsos MORI, 2015, 2016, 2018). Konkret werden Umfrageteilnehmer:innen zu unterschiedlichen Themen in Form von Wissensfragen befragt. Teilgenommen haben an diesen Studien Personen aus 33, 40 bzw. 37 Staaten. Die Zahl der teilnehmenden Personen pro Land und Studie schwankt zwischen 500 und 2.000. Zu erwähnen ist, dass die Teilnehmenden nicht immer ein repräsentatives Abbild der Gesamtbevölkerung darstellen. Die jeweils etwa 1.000 deutschen Teilnehmenden in den PoP-Studien wurden jedoch repräsentativ ausgewählt.

Abbildung 1 stellt dar, wie informiert die Deutschen hinsichtlich einzelner wirtschaftspolitischer Themen sind. Konkret wird gezeigt, wie stark die jeweils durchschnittlich in den PoP-Umfragen abgegebene Antwort von der korrekten Antwort abweicht. Es zeigt sich, dass die Deutschen auf den abgefragten Feldern bemerkenswerte Wissenslücken aufweisen. 2018 wurde z. B. geschätzt, dass die Arbeitslo-

Dr. Sebastian Blesse ist wissenschaftlicher Mitarbeiter am Zentrum für Europäische Wirtschaftsforschung (ZEW) in Mannheim.

Prof. Dr. Friedrich Heinemann leitet dort den Forschungsbereich Unternehmensbesteuerung und Öffentliche Finanzwirtschaft am ZEW und ist außerplanmäßiger Professor für Volkswirtschaftslehre an der Ruprecht-Karls-Universität Heidelberg.

Dr. Tommy Krieger ist wissenschaftlicher Mitarbeiter am ZEW in Mannheim. 
Abbildung 1

Wirtschaftspolitische Faktenkenntnis in Deutschland

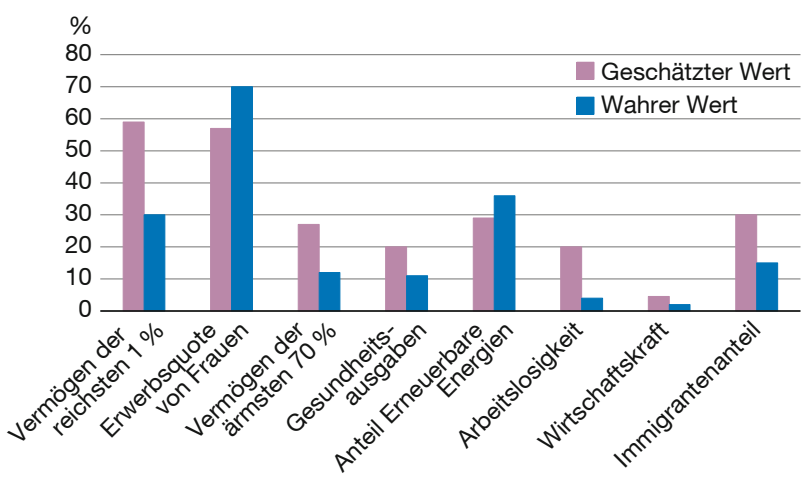

Vergleich der von Deutschen im Rahmen der PoP-Studien auf verschiedene wirtschaftspolitische Wissensfragen durchschnittlich abgegebenen Antwort (pinke Säule) mit dem entsprechenden tatsächlichen Wert (blaue Säule). Eine genaue Beschreibung der genutzten Daten und eine Auflistung der Fragen erfolgt in Blesse et al. (2021).

Quelle: Ipsos MORI (2015, 2016, 2018).

senquote $20 \%$ und der Anteil der im Ausland geborenen Personen $30 \%$ beträgt. Tatsächlich lagen diese Anteile bei ca. $4 \%$ bzw. ca. $15 \%$. Auffällig ist auch, dass Deutsche die Performance ihres Landes tendenziell zu schlecht einschätzen und dass das Ausmaß der wirtschaftspolitischen Uninformiertheit vom Thema abhängt. Besonders stark ausgeprägt ist die Unkenntnis z. B. bei Fragen der Vermögensverteilung. 2015 wurde geschätzt, dass das reichste Prozent der deutschen Bevölkerung 59\% des Gesamtvermögens besitzt. In Wirklichkeit lag dieser Anteil bei $30 \%$.

Für einen Ländervergleich ist ein Gesamtmaß zur wirtschaftspolitischen Uninformiertheit auf Basis der PoP-Studien hilfreich. Um dies zu erstellen, werden alle in Abbildung 1 dargestellten Abweichungen aggregiert (für Details Blesse et al., 2021). Das Gesamtmaß ist aus Gründen der Vergleichbarkeit nur für jene 22 Länder verfügbar, für die für alle acht Wissensfragen Antworten vorliegen. Deutschland nimmt unter diesen Ländern insgesamt den achten Rang ein. Im internationalen Vergleich gehören die Deutschen also nicht zur absoluten Spitzengruppe, sind aber immerhin überdurchschnittlich gut informiert. Werden nur die OECDbzw. EU-Länder miteinander verglichen, dann rangiert Deutschland nur im Mittelfeld.Abbildung 2 zeigt die wirtschaftspolitische Informiertheit in den einzelnen Ländern. Je dunkler der Farbton, desto weniger ist die Bevölkerung informiert. Die am besten informierten Bürger:innen kommen aus Großbritannien, Schweden und den Niederlanden. Auf einem ähnlichen Niveau wie Deutschland befinden sich die USA, Australien und Japan. Besonders hoch ist das Informationsdefizit dagegen in Schwellenländern wie Indien und Brasilien. Innerhalb der EU wird deutlich, dass italienische und spanische Wähler:innen weniger gut informiert sind als Nord- und Zentraleuropäer:innen.
Abbildung 2

Internationaler Vergleich des wirtschaftspolitischen Informationsstands
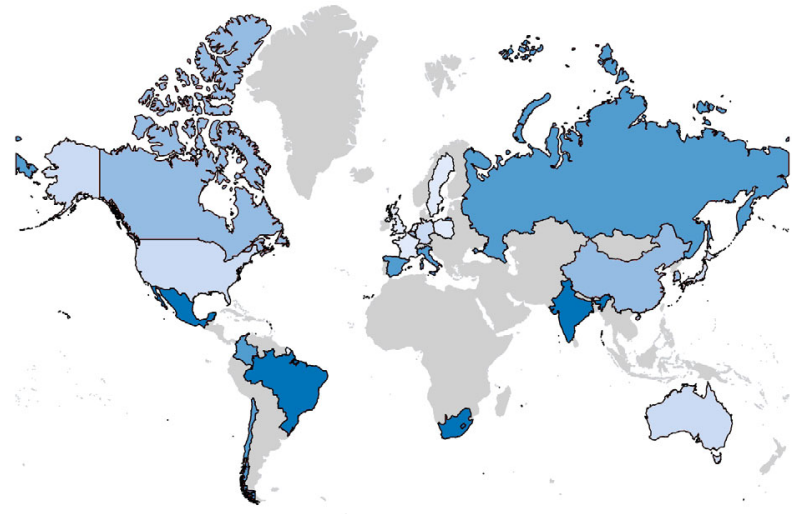

Die Abbildung zeigt die Unterschiede im aggregierten Gesamtindex des wirtschaftspolitischen Informationsstands gemäß der PoP-Daten. Je dunkler der Blauton, desto schlechter der Informationsstand. Für Details zur Konstruktion des Indikators siehe Blesse et al. (2021).

Quelle: Ipsos MORI $(2015,2016,2018)$ und eigene Berechnungen.

\section{Ausgewählte Determinanten des Informationsstands}

\section{Informationen und wirtschaftspolitische Präferenzen}

Wirtschaftspolitische Einstellungen in der Bevölkerung werden durch verschiedene Typen von Determinanten beeinflusst. Es existieren zunächst grundsätzliche Prägungen, die entscheidend für bestimmte Werturteile, wie etwa zur Gleichheitsorientierung und zum Leistungsprinzip, sind. Davon strikt zu unterscheiden sind die unterschiedlichen Wahrnehmungen von institutionellen Rahmenbedingungen, z. B. Regulierungen, Steuern und Transfers, ökonomischen Wirkungszusammenhängen, z.B. durch Steuern ausgelöste Verhaltensreaktionen, und Restriktionen, z. B. für das Budget des Staates und zur Ökologie (Haaland et al., 2021). Während individuelle Prägungen weitgehend nicht durch wirtschaftspolitische Informationen verändert werden, so beeinflusst der Informationsstand die Wahrnehmung von Rahmenbedingungen, Wirkungszusammenhängen und Restriktionen. Konkret kann eine verbesserte Kenntnis um wirtschaftspolitische Fakten und Zusammenhänge auch dann zu einem Überdenken von Positionen führen, wenn die Werturteile unverändert bleiben.

Die Folgen von wirtschaftlicher (Un-)Informiertheit werden zunehmend in Umfrageexperimenten nachgewiesen. Roth et al. (2021) zeigen zum Beispiel, wie sich die Wahrnehmung der staatlichen Budgetrestriktion auswirkt. Personen, die den öffentlichen Schuldenstand unter-/überschätzen, unterstützen höhere/niedrigere Staatsausgaben, da sie jeweils den Finanzierungsspielraum des Staates über-/ unterschätzen. Durch Informationen zum Schuldenstand überdenken diese Menschen ihre Ausgabenpräfenzen, um 
sie in Einklang mit dem bestehenden Finanzierungsspielraum zu bringen (für Befunde ähnlicher Experimente siehe den Überblicksartikel von Haaland et al., 2021). Stantcheva (2021) findet zudem, dass ökonomische Bildung die wirtschaftspolitischen Positionen beeinflusst. Sie untersucht konkret, wie sich Kenntnisse hinsichtlich unterschiedlicher Implikationen der Steuerpolitik auf die Wahrnehmung von Steuern auswirken. Dazu werden Studienteilnehmer:innen Erklärungsvideos gezeigt, die entweder Effizienz- bzw. Verteilungsaspekte oder aber beide Argumente gleichzeitig vermitteln. Hinweise auf Effizienzkosten der Besteuerung erhöhen dabei die Sorgen um mögliche wirtschaftsschädliche Wirkungen höherer Steuern.

Andere experimentelle Studien zeigen, dass sich die Wirkung von Informationen auf wirtschaftspolitische Präferenzen über die Zeit verringern kann. Coppock (2017) etwa beobachtet, dass sich Informationseffekte bereits nach zehn Tagen halbieren und dann für etwa 20 Tage gleichbleiben. Mögliche Gründe für einen schwächer werdenden Informationseffekt sind, dass ein Thema für Bürger:innen mit der Zeit unwichtiger wird oder dass die Informationen nach einer Weile schlicht vergessen werden. Beide Probleme ließen sich durch Maßnahmen zur Steigerung des Interesses an wirtschaftspolitischen Fragestellungen adressieren, denn ein gesteigertes Interesse führt zu mehr eigenen Recherchen. Ein alternativer Ansatz könnte in der Verstetigung des Informationszuflusses liegen.

\section{Politisches (Des-)Interesse und kognitive Fähigkeiten}

Aus Sicht der Theorie der Rationalen Ignoranz ist es für Wähler:innen individuell nicht rational, informierte Wahlentscheidungen zu treffen, da aufwendige Informationsprozesse vor der Wahl zwar eine positive gesellschaftliche Externalität zur Folge haben, dem Individuum aber keinen Ertrag verschaffen (Downs, 1957). Dieser fehlende individuelle Informationsanreiz wird verstärkt, wenn es an intrinsischem politischen Interesse fehlt. In diesem Zusammenhang zeigt z.B. die 18. Shell-Jugendstudie, dass nur $41 \%$ der in Deutschland aufgewachsenen 12- bis 25-Jährigen Interesse an Politik haben (Albert et al., 2019). Dieses Desinteresse ist beunruhigend, da sich in dieser Lebensphase viele grundsätzliche politische Werte und Meinungen herausbilden (Impressionable Years Hypothesis). Bei älteren Menschen ist das Politikinteresse etwas höher (Köcher, 2019). Die Gründe, warum sich (junge) Menschen nur wenig mit Politik befassen, sind vielfältig. Petersen et al. (2013) zeigen z.B., dass Aussagen wie ,in der Politik wird zu viel getrickst und getäuscht" unter den politisch uninteressierten Deutschen große Zustimmung finden. Zudem beobachten diese Autoren, dass sich Desinteressierte von den etablierten Parteien oft nicht repräsentiert fühlen und politische Prozesse als sehr komplex empfinden.
Die kognitiven Fähigkeiten zur Erfassung komplexer Zusammenhänge sind eine Grundvoraussetzung dafür, dass Menschen überhaupt eine Motivation erlangen können, sich näher mit den Feinheiten der (Wirtschafts-)Politik zu befassen. Intellektuelles Unvermögen erzeugt Frustration, auf die Menschen letztlich mit Ablehnung oder sogar gänzlichem Desinteresse reagieren dürften. Geringes Verständnis für Logik und Statistik sowie allgemeine Schwächen bei der Analyse von Texten sind daher weitere potenzielle Ursachen für Uninformiertheit. Dies erklärt, dass der Bildungsstand und die Vertrautheit mit wirtschaftspolitischen Fakten korreliert sind (unter anderem Stantcheva, 2021). Fuster et al. (2020) zeigen, dass Menschen, die ein Grundverständnis für mathematische Zusammenhänge haben, mehr Informationen suchen, bessere Informationsquellen auswählen und neue Informationen besser verarbeiten können.

\section{Medienkonsum}

Während sich eine Mehrheit der Deutschen derzeit immer noch durch das Fernsehen über das aktuelle Tagesgeschehen informiert, dominiert bei den Jüngeren (d. h. den 14- bis 44-Jährigen) das Internet als Informationsquelle (Schneller, 2020). Für den Grad der wirtschaftspolitischen Informiertheit ist die Medienauswahl von hoher Bedeutung. Berichte, die im Radio, Fernsehen oder in Zeitungen veröffentlicht werden, sind eher auf inhaltliche Richtigkeit geprüft worden als Blog-, Twitter- oder Facebook-Posts. Diese Kontrollen verringern das Risiko, dass Falschinformationen (Fake News) in Umlauf geraten und das Faktenwissen verschlechtern oder verzerren (Barrera et al., 2020). Außerdem werden Nutzer:innen von sozialen Medien weniger häufig mit Gegenmeinungen konfrontiert. Dies liegt unter anderem daran, dass Menschen in diesen Medien vor allem Informationen suchen und finden, die den eigenen Erwartungen entsprechen (Nickerson, 1998). Inzwischen gängige Begriffe wie „Internetblasen“ und „digitale Echoräume“ beschreiben die daraus resultierenden Phänomene. Mit der Verwendung von traditionellen Medien können solche Gefahren leichter eingedämmt werden, da die Informationen von anderen Personen (d.h. Journalist:innen) vorsortiert werden. Allerdings wählen auch professionelle Berichterstatter:innen die von ihnen präsentierten Informationen nicht vollkommen neutral aus (Gentzkow und Shapiro, 2010). Auch hier kann es ideologische Verzerrungen und eine Selbstselektion der Mediennutzenden hin zu Zeitungen bzw. TV-Kanälen geben, die in ihrer Ausrichtung der eigenen Überzeugung nahestehen. Dass die Art und Weise, wie die traditionellen Medien Informationen bereitstellen, sowohl einen Einfluss auf die politischen Präferenzen der Wähler:innen als auch auf deren wirtschaftspolitische Informiertheit besitzt, zeigen mehrere Studien (z.B. DellaVigna und Kaplan, 2007). Dennoch ist davon auszugehen, dass die Abschottung gegen konträre Sichtweisen in den digitalen Räumen deutlich 
stärker erfolgen kann als in den konventionellen analogen Medien, denn diese sind ihrem Selbstverständnis nach auf ein Mindestmaß an pluraler Berichterstattung ausgerichtet.

\section{Handlungsempfehlungen}

Die folgenden Maßnahmen könnten unter Bezug auf die zuvor skizzierten Erkenntnisse einen Beitrag zur Verbesserung der wirtschaftspolitischen Informiertheit in Deutschland leisten. Entwickelt wurden diese Vorschläge unter drei Gesichtspunkten: Erstens sollten entsprechende Lösungsansätze zielgruppenorientiert sein. Während es bei jungen Leuten vor allem darum geht, Interesse für wirtschaftspolitische Themen zu entwickeln und Kernkompetenzen zu fördern, müssen ältere Wähler:innen effizienter und regelmäßiger an wirtschaftspolitisch relevante Informationen gelangen. Zweitens wird das in Deutschland aufgrund der vielfältigen öffentlich-rechtlichen Medien und angewandten Forschungseinrichtungen existierende Potenzial für die Bereitstellung ausgewogener Informationen bisher nicht ausreichend ausgeschöpft. Drittens lässt sich die wirtschaftspolitische Informiertheit in Deutschland nur dann nachhaltig steigern, wenn mehrere Beteiligte (Politik, Medien, Wissenschaft) ihre Beiträge umfassend und gemeinsam leisten.

\section{Anreize für Wissenstransfer schaffen}

Einrichtungen der universitären und angewandten Wirtschaftsforschung bergen bislang ungenutzte Potenziale zur Hebung der ökonomischen Kompetenz der Bevölkerung. Der Vorschlag „Leistungspunkte für Wissenstransfer" möchte diese Potenziale besser ausschöpfen. Dazu sollen junge Wissenschaftler:innen stärkere Anreize zur Beteiligung am Wissenstransfer bekommen. Der Ausgangspunkt dieses Vorschlags ist die Beobachtung, dass sich Promovierende und Post-Docs trotz intrinsischen Interesses am Wissenstransfer oft schwertun, bei entsprechenden Formaten mitzuwirken. Der Grund dafür ist, dass ein solches Engagement auf dem wissenschaftlichen Arbeitsmarkt kaum belohnt wird. Prinzipiell kann sich eine solche Aktivität sogar negativ auf die eigenen Karrierechancen auswirken, da sie Arbeitszeit in Anspruch nimmt, die andernfalls in die Verwirklichung von Forschungsvorhaben gesteckt werden könnte. Um dem entgegenzuwirken, könnten Anreize dafür geschaffen werden. Vorstellbar wäre dabei z. B., dass Promovierende im Rahmen ihres Promotionsstudiums nicht nur Leistungspunkte für Vorträge in Fachseminaren erhalten, sondern auch für Initiativen, die primär dem Zweck des Wissenstransfers dienen. Bisher ist dies meist nicht der Fall. Zur Umsetzung dieser Maßnahme ist ein Umdenken bei den deutschen Universitäten und Forschungsinstituten notwendig, denn bisher spielt der Aspekt des Wissenstransfers an Laien in der wissenschaftlichen Ausbildung eine untergeordnete Rolle.

\section{Verständnis für Kausalität schärfen}

Die empirische Mikroökonomie hat sich in den vergangenen 20 Jahren erheblich weiterentwickelt. Während sich früher die meisten Volkswirte mit relativ einfachen Analyseverfahren zufriedengaben und ihre Schlüsse auf Grundlage robuster Korrelationen zogen, sehen sie sich heute methodisch auf einer Linie mit Naturwissenschaftler:innen, da sie nun auch mithilfe von Experimenten versuchen, kausale Zusammenhänge aufzudecken. Der diesjährige Nobelpreisträger Joshua Angrist und Jörn-Steffen Pischke haben diesen Wandel als „Credibility Revolution“ bezeichnet und dargestellt, wie wichtig das Streben nach Kausalität ist und wie irreführend die Interpretation von Korrelationen sein kann (Angrist und Pischke, 2010).

Hier wäre es wichtig, das Bewusstsein für den Unterschied zwischen Korrelation und Kausalität insbesondere auch im wirtschafts- und sozialwissenschaftlichen Bereich bereits bei Schüler:innen zu erhöhen. Dabei geht es nicht darum, dass diese lernen, komplexe empirische Methoden anzuwenden. Vielmehr sollten sie anhand einfacher wirtschaftspolitischer Beispiele verstehen, dass selbst robuste Korrelationen nicht mit Kausalität gleichgesetzt werden können. Die Fähigkeit, Korrelation und Kausalität unterscheiden zu können, führt mittel- und langfristig zu mehr wirtschaftspolitischer Informiertheit, da junge Menschen weniger geneigt sein werden, einfachen Aussagen Glauben zu schenken.

\section{Jungen Menschen den Dialog mit Forschenden erleichtern}

Geringes Interesse ist gerade bei jungen Menschen ein Hauptgrund für wirtschaftspolitische Informationsdefizite. Dieses Problem gilt es anzugehen. Empfehlenswert sind dafür z. B. Maßnahmen, die den Austausch zwischen Schulen und Forschungseinrichtungen intensivieren. Hier liegt im Bereich der deutschen Wirtschaftsforschungsinstitute bisher nicht vollständig ausgeschöpftes Potenzial, denn in diesen arbeiten viele Promovierende und junge Post-Docs, die in ihrem Alter den Schüler:innen noch vergleichsweise nahe sind. Der Vorschlag „Book a Scientist for Teens“ beinhaltet, das von der Leibniz-Gemeinschaft vor wenigen Jahren ins Leben gerufene Wissenschaftstransferformat „Book a Scientist“ als Vorbild für ein speziell auf junge Menschen ausgerichtetes Modell zu nutzen. Das Grundprinzip dieses Formats besteht darin, dass sich interessierte Bürger:innen Gesprächstermine bei Mitarbeiter:innen der Leibniz-Institute buchen können, um diese über deren Forschung zu befragen. Diesem Ansatz folgend, könnten bei „Book a Scientist for Teens" Schulklassen die Möglichkeit erhalten, Wissenschaftler:innen einzuladen, um mit diesen zu diskutieren. 
Wirtschaftsrelevante Medienkompetenz stärken

Die Auswahl von und der Umgang mit Medien ist eine wichtige Determinante für wirtschaftspolitische Uninformiertheit. Dies gilt besonders für jüngere Menschen, da sie soziale Medien wesentlich häufiger nutzen als ältere Menschen und dadurch öfter mit Informationen aus ungeprüften Quellen in Berührung kommen. Daher liegt es nahe, dass zur Reduzierung der Uninformiertheit die digitale Medienkompetenz von früh auf intensiv gefördert werden sollte. Die Förderung proaktiven Denkens (Pennycook et al., 2021) und digitale Alphabetisierungskampagnen (Guess et al., 2020) können die Qualität der online konsumierten Nachrichten verbessern und Nutzer:innen von sozialen Medien weniger anfällig für Fehlinformationen machen. Roozenbeek und van der Linden (2019) zeigen zudem auf, dass „Fake-News-Spiele“ helfen, unglaubwürdige Schlagzeilen leichter zu identifizieren. Die allgemeinbildenden Schulen haben in den vergangenen Jahren entsprechend den unterschiedlichen Akzentsetzungen in den Bildungsplänen der Länder bereits in erheblichem Maße Initiativen zur Verbesserung der digitalen Kompetenz der Schüler:innen unternommen. Diese Bemühungen sind verdienstvoll und der eingeschlagene Weg sollte fortgesetzt werden. In Zukunft sollte dabei noch mehr die Fähigkeit gefördert werden, verlässliche (wirtschafts-)politische Informationen zu finden. Erwägenswert ist, diesen Kompetenzerwerb durch besondere Zertifikate wie einen "Internet-Führerschein“ stärker sichtbar zu machen, um dadurch weitere Anreize bei den Schüler:innen zu schaffen. Eine Orientierung für ein solches Zertifikat bietet z.B. der Internetführerschein für Kinder des gemeinnützigen Anbieters Internet-ABC.

Faktencheck für Wahlprogramme einführen

Wegen der oft vielfältigen Wirkungskanäle von wirtschaftspolitischen Reformen und der meist nur skizzenartigen Beschreibung in Wahlprogrammen ist es oft schwer einzuschätzen, wie sich eine konkrete Maßnahme auf die Wähler:innen selbst und die wirtschaftliche Gesamtentwicklung auswirkt. Empfehlenswert sind daher Ansätze, welche die positiven und negativen Folgen bestimmter Reformvorhaben in neutraler Form zusammenfassen. Ein Blick in die Niederlande zeigt, wie dies gelingen kann. Dort reichen wahlwerbende Parteien ihre Wahlprogramme in einer weitestgehend standardisierten Form bei einem unabhängigen staatlichen Institut, dem Centraal Planbureau (CPB), ein. Das CPB analysiert die Wahlprogramme hinsichtlich ihrer Konsistenz, ihrer Auswirkungen auf verschiedene makroökonomische und fiskalpolitische Größen sowie deren Verteilungseffekte. Zwei Monate vor der Wahl veröffentlicht das CPB seine Resultate (Jacobs et al., 2017). Das in den Niederlanden angewandte Verfahren führt dazu, dass Parteien relativ konkrete Wahlvorschläge unterbreiten und in deren Programmen nur wenig Raum für leere Wahlversprechen bleibt. Des Weiteren stellt die Analyse des CPB auch eine klare und transparente Informationsgrundlage dar, da sie anhand der Analyseergebnisse Reformen mit konkreten wirtschaftlichen Folgen verknüpfen können. Entscheidend für die Glaubwürdigkeit wäre ein unabhängiger Analyseprozess durch „Wahlprogramm-Referees“. Denkbar wäre hier der Einbezug des Bundesrechnungshofs, des Sachverständigenrats zur Begutachtung der gesamtwirtschaftlichen Entwicklung oder eine Gemeinschaftsanalyse der Wirtschaftsforschungsinstitute. Die Qualitätssicherung müsste durch Peer-Reviews aus der Wissenschaft erfolgen, um politische Einflussnahme auszuschließen.

\section{Restriktionen veranschaulichen}

Ein zentrales Problem in der Meinungsbildung über Wirtschafts- und Finanzpolitik ist, dass eine rationale Entscheidung eine vollständige Kosten-Nutzen-Analyse der entsprechenden staatlichen Maßnahmen umfassen und alle bestehenden Restriktionen berücksichtigen sollte. Zusätzliche Staatsausgaben sind z. B. durch Steuern oder Schulden zu finanzieren, wobei die Schuldenfinanzierung letztlich bei Geltung der intertemporalen Budgetrestriktion des Staates einer Verschiebung der Steuerlast in die Zukunft gleichkommt. Zu ineffizienten Entscheidungen kommt es dann, wenn Wähler:innen zwar den Nutzen der Staatsausgaben wahrnehmen, aber nicht mit den Kosten und den entsprechenden Finanzierungskonsequenzen konfrontiert werden. Dann werden auch solche Projekte realisiert, bei denen die (Grenz-)Kosten den (Grenz-)Nutzen übersteigen. Die Fachliteratur bestätigt, dass die Bereitstellung von Informationen zur staatlichen Budgetrestriktion (z.B. durch den Stand der Staatsschulden wie in Roth et al., 2021) die Nachfrage nach zusätzlichen Staatsausgaben beeinflussen kann. Allerdings sind diese Informationen nur Wenigen präsent. In Wahlkampfsituationen haben Parteien einen Anreiz, über die Kosten ihrer Ausgabenpläne zu schweigen, weil Kostentransparenz die Zustimmung verringern kann. Schon die Wahlprogramm-Referees würden hier teilweise Abhilfe schaffen. Um dieses Problem permanent, d.h. auch außerhalb von Wahlkampfzeiten, zu adressieren, sollten die öffentlich-rechtlichen Medien regelmäßig über objektive finanzpolitische Restriktionen berichten. So wäre es denkbar, in oder kurz vor einem täglichen Nachrichtenformat über den „Fakt des Tages“ zu berichten. Beispiele wären die Pro-Kopf-Staatsverschuldung, Ausgaben für bestimmte Politikbereiche oder andere wirtschaftspolitische Indikatoren, die direkt oder indirekt Informationen über Knappheiten und Reformherausforderungen vermitteln. Der „Fakt des Tages" sollte nicht nur über finanzpolitische Restriktionen berichten, sondern auch über andere Arten von Knappheiten, die für eine rationale Wirtschaftspolitik berücksichtigt werden müssen (z. B. ökologische Restriktionen). 


\section{Ökonomische Rückwirkungen aufzeigen}

Faktenchecks sind zu einem festen Bestandteil medialer Arbeit geworden. Dies ist zu begrüßen, da damit klare Falschaussagen aufgedeckt werden können und politische Akteur:innen schon im Vorfeld gezwungen werden, sauberer zu arbeiten, weil sie ansonsten mit Reputationsschäden rechnen müssen. Die Überprüfung von Tatsachen-Aussagen, die ja auch durch die zuvor beschriebene Initiative „Fakt des Tages“ unterstützt würde, kann aber ein weiteres Ziel für die kompetente Beurteilung von Wirtschaftspolitik nicht erreichen, nämlich das Bewusstsein für marktbezogene Rückwirkungen von Politikmaßnahmen. Beispiele sind etwa: In welcher Weise kann eine Preisregulierung von Mieten zu Veränderungen des Wohnungsangebots führen, oder wie stark könnten Investitionen durch einen Anstieg von Gewinn- oder Vermögensteuern in Mitleidenschaft gezogen werden? Menschen neigen dazu, nur auf die unmittelbaren Primäreffekte einer Reform zu schauen, z.B. welcher Einnahmeeffekt durch die Erhöhung einer Steuer zugunsten des Staates erzielt wird. Die nachgelagerten Fragen, z.B. wie sich eine Preisregulierung auf Investitionen auswirkt, werden dagegen oftmals ignoriert (Dal Bó et al., 2018). Daraus ergibt sich eine Präferenz für "einfache Lösungen“, die aufgrund ihrer nur vordergründigen Wirkungsanalyse ihre eigentlichen Ziele möglicherweise nicht (umfassend) erreichen können. Um das Bewusstsein für solche Zweitrundeneffekte zu schärfen, sollten Faktenchecks in den Medien durch „Folgenchecks" ergänzt werden. Hier besteht allerdings die Schwierigkeit, dass auch in der empirischen Forschung nicht unbedingt Einigkeit über Existenz und Ausmaß der indirekten Wirkungen einer Maßnahme bestehen. Ein Lösungsansatz könnte darin bestehen, „Meta-Analysen“ mehr Aufmerksamkeit zu schenken, denn diese berichten umfassend über die Erkenntnisse der Fachliteratur. Der Vorteil von Meta-Studien ist, dass sie die komplette Bandbreite empirischer Effekte zu einer bestimmten Fragestellung aufzeigen und damit auch vermitteln können, wie groß der wissenschaftliche Konsens ist. Erreicht werden kann dies z. B. durch Formulierungen wie: „Die Wissenschaft schätzt, dass die Erhöhung der Steuern auf Direktinvestitionen zu einer Verringerung der Investitionen um $X$ bis $Y$ Prozent führen würde." Dieser Folgencheck für unterschiedliche Wirtschaftsthemen könnte wiederum durch öffentlich-rechtliche Medien in Zusammenarbeit mit öffentlich finanzierten Wirtschaftsforschungsinstituten erstellt werden.

\section{Literatur}

Albert, M., K. Hurrelmann und G. Quenzel (2019), Jugend 2019: Eine Generation meldet sich zu Wort, 18. Shell Jugendstudie.

Angrist, J. D. und J. S. Pischke (2010), The credibility revolution in empirical economics: How better research design is taking the con out of econometrics, Journal of Economic Perspectives, 24(2), 3-30.

Barrea, O., S. Guriev, E. Henry und E. Zhuravskaya (2020), Facts, alternative facts, and fact checking in times of post-truth politics, Journal of Public Economics, 1872(1), 104123.

Blesse, S., F. Heinemann und T. Krieger (2021), Informationsdefizite als Hindernis rationaler Wirtschaftspolitik: Ausmaß, Ursachen und Gegenstrategien. ZEW-Gutachten mit Unterstützung der Brigitte Strube Stiftung.

Caplan, B. (2007), The myth of the rational voter, Princeton University Press.

Coppock, A. (2017), The persistence of survey experimental treatment effects, https://alexander coppock.com/coppock_2017b.pdf (15. Oktober 2021).

Dal Bó, E., P. Dal Bó und E. Eyster (2018), The demand for bad policy when voters underappreciate equilibrium effects, Review of Economic Studies, 85(2), 964-998.

DellaVigna, S. und E. Kaplan (2007), The Fox News effect: Media bias and voting, Quarterly Journal of Economics, 122(3), 1187-1234.

Downs, A. (1957), An economic theory of political action in a democracy, Journal of Political Economy, 65(2), 135-150.

Fuster, A., R. Perez-Truglia, M. Wiederholt und B. Zafar (2020), Expectations with endogenous information acquisition: An experimental investigation, Review of Economics and Statistics, im Erscheinen.

Gentzkow, M. und J. Shapiro (2010), What drives media slant? Evidence from US daily newspapers, Econometrica, 78(1), 35-71.

Guess, A. M., M. Lerner, B. Lyons, J. M. Montgomery, B. Nyhan, J. Reifler und N. Sircar (2020), A digital media literacy intervention increases discernment between mainstream and false news in the United States and India Proceedings of the National Academy of Sciences, 117(27), 15536-15545.

Haaland, I., C. Roth und J. Wohlfart (2021), Designing information provision experiments, Journal of Economic Literature, im Erscheinen.

Ipsos MORI (2015), Perils of perception 2015.

Ipsos MORI (2016), Perils of perception 2016.

Ipsos MORI (2018), Perils of perception 2018.

Jacobs, B., E. L. W. Jongen und F. T. Zoutman (2017), Revealed social preferences of Dutch political parties, Journal of Public Economics, 156(1), 81-100.

Köcher, R. (2019), Eine neue politisierte junge Generation?, Allensbacher Markt- und Werbeträgeranalyse.

Nickerson, R. S. (1998), Confirmation bias: A ubiquitous phenomenon in many guises, Review of General Psychology, 2(2), 175-220.

Pennycook, G., Z. Epstein, M. Mosleh, A. A. Arechar, D. Eckles und D. G. Rand (2021), Shifting attention to accuracy can reduce misinformation online, Nature, 592(7855), 590-595.

Petersen, T., D. Hierlemann, R. B. Vehrkamp und C. Wratil (2013), Gespaltene Demokratie: Politische Partizipation und Demokratiezufriedenheit vor der Bundestagswahl 2013, Bertelsmann Stiftung.

Roozenbeek, J. und S. van der Linden (2019), Fake news game confers psychological resistance against online misinformation, Palgrave Communications, 5(1), 1-10.

Roth, C., S. Settele und J. Wohlfart (2021), Beliefs about public debt and the demand for government spending. Journal of Econometrics, im Erscheinen.

Schneller, J. (2020), Entwicklungstrends in der Mediennutzung der Bevölkerung und die aktuelle Situation im Herbst 2020.

Stantcheva, S. (2021), Understanding tax policy: How do people reason?, Quarterly Journal of Economics, 136(4), 2309-2369.

Title: Economic Disinformation - Causes and Recommendations for Action

Abstract: This articles exploits the results of several Perils-of-Perception studies to investigate how informed the German population is about key economic facts. There are significant misperceptions and Germany ranks in the middle of the group of advanced economies. Our overview briefly sketches different possible explanations for the relatively low factual knowledge of the German society. We propose seven measures that may help to improve the level of economic literacy in Germany, both among young people as well as in the broader segments of the population. Our measures address different stakeholders, such as policymakers, the media and the academic community.

JEL Classification: $D 83$ 